\title{
Measuring Polariscopes
}

This content has been downloaded from IOPscience. Please scroll down to see the full text. 1879 Proc. Phys. Soc. London 3112

(http://iopscience.iop.org/1478-7814/3/1/317)

View the table of contents for this issue, or go to the journal homepage for more

Download details:

IP Address: 128.119.168.112

This content was downloaded on 06/09/2015 at 15:19

Please note that terms and conditions apply. 
differs greatly from that in the dispersion-spectrum, and that in the dispersion-spectrum the great calorific intensity of the red rays, and therefore in all probability of the invisible rays beyond them, is due to the action of the prism in concentrating these rays upon the face of the thermopile. The intensity of the heat in the different portions of the normal spectrum, except in the case of Lamansky's measurements with the flintglass prism, apparently varies but little through a considerable space; and this affords some support to Dr. Draper's hypothesis, that every colour ought to have the same heating effect.

After I had finished these calculations, I found that $G$. Lundquist had investigated (Pogg. Ann. clv. p. 146), from Lamansky's measurements, the distribution of heat in the normal spectrum, and had shown that it differed greatly from the distribution in the dispersion-spectrum-the maximum intensity in the case of the flint-glass prism being near $D$, and in the rock-salt prism near E. He also found from Tyndall's measurements of the heat of the electric-light spectrum, that in the normal spectrum the maximum was near $A$.

Lundquist arrived at these results by a mathematical prucess, based on the same general principles as the graphical one I have employed.

\section{Measuring Polariscopes. By Professor W. Grylls Adams, M.A., F.R.S.* [Plate XIV.]}

Some four years ago the description of a new measuring polariscope was communicated by the author to the Physical Society (see 'Proceedings,' vol. i. p. 152), in which the advantages gained are an extensive field of view combined with accurate means of measuring the rings and the separation of the optic axes in biaxal crystals. The peculiarity of the instrument consists in the arrangement of the two central lenses, one on each side of the crystal. These two lenses are planoconvex, very nearly hemispheres, and, with their flat surfaces inwards, form the two sides of a box to hold the crystal immersed in oil or a liquid; they are so placed that their convex

* Read June 28. 
Proc.Phys. Soc.Vol. 3. Pl.XIV.
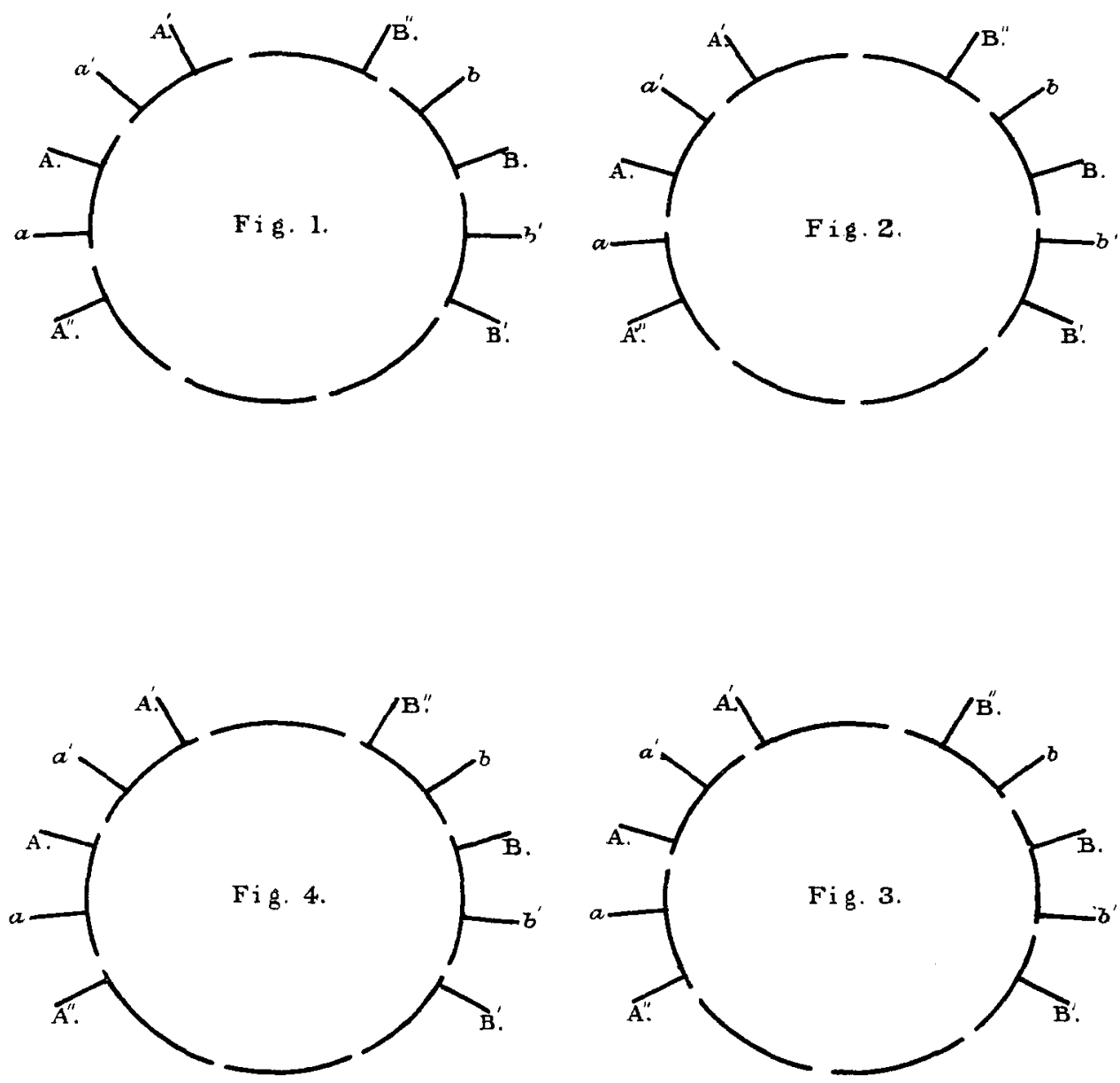
surfaces form portions of the same spherical surface. The crystal is placed in the box at the centre of curvature of the spherical surfaces of the two lenses.

Two instruments have since been made on this principle with certain important modifications. In one, made by $\mathrm{Mr}$. Tisley for horizontal projection (Plate XIV. fig. 1), the polarizer is a Nicol's prism capable of giving a clear parallel beam of polarized light $2 \frac{1}{2}$ inches in diameter; the middle portion of the instrument ( $i$.e. the box with the two equal central lenses for its two opposite sides) has an opening at the top, into which the crystal to be measured is inserted, and is adjusted to its right position by a cup-and-socket motion. When the angle between the optic axes is to be measured, the instrument is placed with its axis horizontal, the crystal is placed with the plane of the optic axis vertical, the box and crystal together are then turned about a horizontal axis at right angles to the direction of the axis of the instrument, $i . e$. at right angles to the plane of the optic axes: thus either of the optic axes of the crystal may be made to coincide with the centre of the field of view, where the spider-lines cross one another, the angle through which the box is turned being measured to minutes by means of a circle attached to it and a vernier attached to the fixed stand supporting the instrument.

A table-polariscope on the same principle has been made by Herr Schneider of Vienna, into which several important modifications have been introduced. A section and view of the instrument are shown in figs. 2 and 3.

The light falls on a plane mirror $A$, and is reflected into the instrument (which is placed with its axis vertical) through the first lens $B$, which is fixed on the tube in which the polarizer $\mathrm{C}$ is placed.

D, E, F, G, and $\mathrm{H}$ (fig. 2) are lenses through which the light passes; and $\mathrm{K}$ is a Nicol's prism (the analyzer). This part of the instrument forms a complete table-polariscope of considerable range. The Nicol's prism $\mathrm{C}$ and the lenses are supported each by means of two screws (shown in fig. 3), which may be moved upwards or downwards in two slots, and the lenses fixed in their proper positions. Between the plano-convex lenses $\mathrm{E}$ and $F$ are the two central lenses $L, M$, two portions of a sphere. 
between which the crystal to be measured is placed. The crystal should be immersed in oil. The setting of the lens $L$ has a screw on its surface which fits into a screw in the setting of the other lens $M$, the arm supporting the lenses being a flat piece of metal one sixth of an inch thick, which is placed between them before they are screwed together. This arm, I, is supported by a stout crosspiece $T$, in the form of the arc of a circle which has its centre at the centre of curvature of the two lenses: the arc subtends nearly a right angle at the centre. It passes through and is supported by two guides $\mathrm{P}, \mathrm{Q}$, and has on its outer surface a rack which works with a small toothed wheel turned by the milled head $\mathrm{N}$.

On the upper face of the arm I is a train of toothed wheels, the setting of the lens $M$ being provided with teeth, so that, by means of the milled head $S$ on the outer wheel, the lenses and crystal may be turned about the axis of the instrument.

The guides $P$ and $Q$ are attached to the face of a vertical circle which may be fastened by screws to a circular graduated rim $R$; this rim is supported on and turns upon another fixed vertical circle, which is provided with verniers, so that the angle through which the rotating circle is turned can be measured to one minute. The fixed circle is fastened to and carried by the piece of tubing holding the two lenses $E$ and $F$, which is supported between the two pieces of tubing carrying the upper and lower lenses.

In this instrument the crystal can be turned about each of three axes which are mutually at right angles to one another.

I. By turning the vertical circle and tubing to which it is attached, the crystal is turned about the vertical axis of the instrument.

II. By turning the train of wheelwork the crystal can also be turned about an axis passing centrically through the central lenses, and the plane of its optic axes may be brought into the desired position for measurement.

III. By turning the arc $\mathrm{T}$ hy means of the milled head $\mathrm{N}$, the axis of the central lenses may be made to coincide with the axis of the instrument; the plane of the optic axes is then parallel to the vertical fixed circle, the crystal being turned by this motion about an axis bisecting the obtuse angle between the optic axes. 
IV. By turning the graduated circle with the arc $\mathrm{T}$ and the arm I attached to it on the face of the fixed vertical circle (i.e. round a horizontal axis perpendicular to the plane of the optic axes), and reading the verniers on both sides of the circle, the angles between the directions of the optic axes may be accurately measured.

\section{A Mode of producing Arago's Rotation.}

\section{By Walter Baily, M.A.*}

[Plates XV. \& XVI.]

ARAGo's method of producing rotation in a copper disk consists of suspending it by its centre so as to make it lie horizontally above the poles of a horseshoe magnet, and then rotating the magnet about a vertical axis. The rotation of the disk is due to that of the magnetic field in which it is suspended; and we should expect that if a similar motion of the field could be produced by any other means, the result would be a similar motion of the disk.

Possibly the rotation of the magnet may be the only practicable way of producing a uniform rotation of the field; but it will be shown in this paper that the disk can be made to rotate by an intermittent rotation of the field effected by means of electromagnets.

Suppose two magnetic poles to be below a plane sheet of a conducting substance capable of moving in its own plane. Each pole may be regarded as a small circular current parallel to the disk. The currents will be in the same or different directions according as the poles are of the same or different names. We will examine the effect of a change in the strength of either of the poles, in giving the sheet a tendency to move.

There are four cases ; viz.-

1. Poles alike. One increasing.

2. " " One diminishing.

3. Poles unlike. One increasing.

4. ", " One diminishing.

In case (1) the increasing pole induces in the portion of the sheet opposite to itself a circular current opposite in direction 